\title{
Uma Proposta de Modelo de Atendimento de Demandas Destinadas a Setores de Inteligência, Pesquisa e Análise de Instituições Públicas Brasileiras ${ }^{1}$
}

Gleidson Sobreira Leite ${ }^{2}$

\section{RESUMO}

\begin{abstract}
A evolução da tecnologia da informação não só proporcionou impacto na vida da sociedade, mas, também, na evolução e forma como os criminosos vêm atuando. Nesse contexto, instituições públicas vêm adotando medidas para combater o crime organizado no Brasil, no qual, entre ações existentes, podemos citar a instalação dos Laboratórios de Combate à Lavagem de Dinheiro, e criação de setores de inteligência, pesquisa e análise. Apesar de bons resultados apresentados por essas medidas, infelizmente a demanda de solicitações é alta, o que acarretam limitações quanto a seu atendimento. Este trabalho propõe disponibilizar uma proposta complementar de modelo de atendimento de demandas encaminhadas a esses setores e laboratórios, com o intuito de contribuir com a agilidade no atendimento por meio da automatização de procedimentos operacionais, com redução de custos para as instituições, e redução de tempo para a realização de diversas atividades de investigação e análise.
\end{abstract}

Palavras-chave: Corrupção. Lavagem de dinheiro. Sistemas de Informação.

1 Data de recebimento: 02/06/2018. Data de aceite: 18/06/2018.

2 Servidor Público. Analista Ministerial de Tecnologia da Informação do Ministério Público do Estado do Ceará lotado no Grupo de Atuação Especial contra o Crime Organizado (GAECO). Pós-Graduação em Segurança da Informação da Universidade Estácio de Sá. Mestre em Informática Aplicada pela Universidade de Fortaleza. Doutorando em Informática Aplicada pela Universidade de Fortaleza. Email gleidson.sleite@gmail.com 


\section{INTRODUÇÃO}

Nos últimos anos, temas referentes ao mau uso dos recursos públicos e corrupção têm se manifestado constantemente nas manchetes, assim como a repercussão da indignação dos brasileiros, que vem também se demonstrando crescente por meio de manifestações públicas, ou em mídias on-line.Sendo a corrupção, conforme citado por Paiva (2017), um comportamento racional e de interesse privado de pessoas, que utilizam seu poder de discrição para alocação de recursos para si ou terceiros em prol de recompensas, de acordo com Saadi (2017), o desvio de recursos públicos, ou gestão criminosa dos mesmos, causa impacto negativo no desenvolvimento do país, acarretando no incremento da desigualdade social, danos na manutenção e prestação de serviços essenciais aos cidadãos, e impactos negativos no desenvolvimento de obras públicas e na geração de empregos e riquezas.

Diante desse cenário, uma atuação governamental comprometida com o desenvolvimento, legalidade e probidade é essencial para, não só visar à prestação de serviços eficientes em prol da sociedade, mas, também, para o enfrentamento da criminalidade, corrupção, entre outros.

Conforme pesquisa desenvolvida pela Transparência Internacional (2017), organização não governamental que visa a enfrentar a corrupção em âmbito global por meio de ações que congregam governos, empresas e sociedade civil, o índice de percepção da corrupção entre os brasileiros colocou o país na 79ª posição entre os 176 países pesquisados, no qual percebeu-se também em pesquisa realizada em 2013, que os partidos políticos foram identificados como as instituições mais corruptas, imediatamente seguidas pelo Congresso Nacional e pela Polícia (TRANSPARÊNCIA INTERNACIONAL, 2013).

Ou seja, cada vez mais se torna essencial a atuação eficiente de órgãos voltados à fiscalização, controle e investigação da gestão e 
uso de recursos públicos, de forma a minimizar os danos causados à sociedade e ao país.

Com o passar do tempo, a evolução e influência da tecnologia da informação tem impactado não só campos técnicos, acadêmicos ou empresariais, mas, também, toda a sociedade, na medida em que usamos cada vez mais serviços on-line para acessar informações, realizar trabalhos, entre outros, permitindo que a cada dia a tecnologia venha se consolidando como um dos principais meios de comunicação, de relacionamentos e negócio.

Diante da facilidade e comodidade proporcionada pela mesma, sua utilização também contribui, exponencialmente, para o incremento e expansão da atuação de instituições públicas em benefício do interesse da sociedade, como por exemplo o Ministério Público, que foi incumbido, conforme Constituição Federal de 1988, da defesa da ordem jurídica, do regime democrático e dos interesses sociais e individuais indisponíveis (BRASIL, 1988).

Dessa forma, essas instituições públicas têm implantado diversas ações e estratégias voltadas ao incremento de resultados, que visam minimizar as consequências advindas do crime organizado, mal uso de recursos públicos, corrupção, lavagem de dinheiro, entre outros, como, por exemplo, a implantação de setores especializados como os de inteligência, pesquisa e análise, ou então laboratórios de combate à lavagem de dinheiro.

Apesar das diversas iniciativas implantadas nas instituições, infelizmente a demanda referente ao combate à corrupção, lavagem de dinheiro e demais crimes é alta.

Citando como exemplo os Ministérios Públicos, ocorreram implantações de laboratórios de tecnologia contra a lavagem de dinheiro e setores de inteligência, pesquisa e análise para dar suporte e atendimento das demandas especializadas das promotorias e procuradorias, que atendem a sociedade, assim como para órgãos de investigação como, por exemplo, Grupo de Atuação Especial de 
Combate às Organizações Criminosas (GAECO), ou Núcleos de Investigação Criminal (NUINC) (MINISTÉRIO DA JUSTIÇA, 2018).

Porém, apesar dos excelentes resultados obtidos com o auxílio desses laboratórios e setores especializados, como por exemplo, a identificação pela rede brasileira de laboratórios de tecnologia contra a lavagem de dinheiro, conforme a Secretaria de Segurança Pública do Maranhão, de cerca de 44,3 bilhões de reais originados de atividades suspeitas de irregularidades nos últimos 11 anos, com o tempo, o alto número de demandas a esses setores acarretou em limitações quanto a seu atendimento (SECRETARIA DE SEGURANÇA PÚBLICA DO MARANHÃO, 2018).

Isso ocorre pelo fato de que o aparato tecnológico e equipe com a devida expertise estar centralizada e limitada em setores (fora a limitação quantitativa de recursos humanos), nos quais os órgãos de investigação, e demais órgãos e promotorias, para o caso do exemplo de Ministérios Públicos, acabam encaminhando um alto volume de demandas para os laboratórios em virtude das limitações de conhecimento, expertise tecnológica ou especializada, entre outras, existentes nos próprios órgãos e promotorias.

Ou seja, os recursos tecnológicos, assim como humanos com as devidas especializações técnicas (economia, contabilidade, análise bancária, entre outros), são limitados, o que acarretou em centralização das demandas de inteligência, pesquisa e análises nesses setores e laboratórios especializados, onde, com o tempo, surgiu a necessidade de definição de critérios de aceitação dessas demandas.

Assim, apesar dos excelentes resultados advindos com a criação desses setores, com o tempo, tiveram que definir linhas de cortes para aceitação de suas demandas devido ao alto volume, surgindo assim a necessidade de criação de um modelo complementar de atendimento das mesmas, proposta objeto deste trabalho, com o intuito de possibilitar o incremento de resultados (atendimentos), redução de tempo e custo para a realização das atividades, assim 
como a possibilidade de expansão do acesso a recursos tecnológicos especializados aos próprios demandantes.

Este trabalho também pretende ser uma contribuição aos estudos acerca do combate à corrupção e lavagem do dinheiro, mediante a apresentação da proposta composta por soluções tecnológicas, que visam à redução do volume de demandas aos laboratórios e setores especializados, por via da aplicação de uma camada intermediária, composta por sistemas tecnológicos automatizados, entre os demandantes e esses setores.

Neste trabalho, também são apresentados resultados e pontos positivos de uma experiência de uso, conforme proposta modelo objeto deste trabalho, composta pela implantação de um sistema informatizado de investigação em um Ministério Público Estadual.

Este artigo, organizados em seções, procura apresentar em linhas gerais: metodologia de pesquisa do trabalho (Seção 2); ações e estratégias já implantadas atualmente pelas instituições públicas brasileiras no combate à corrupção e lavagem de dinheiro (Seção 3); uma proposta de modelo complementar as soluções atualmente implantadas por essas instituições (Seção 4); uma experiência de uso com respectivos resultados (Seção 5); e finalmente, as considerações finais e possibilidades de trabalhos futuros (Seção 6).

\section{METODOLOGIA DE PESQUISA}

Para a realização do objetivo deste trabalho, realizaram-se três ações específicas. Foram elas:

1) Pesquisa bibliográfica sobre as principais ações e estratégias adotadas pelas instituições públicas no tema relacionado ao combate à corrupção e a lavagem de dinheiro, onde, das principais ações/estratégias, foram selecionadas duas (Laboratórios de Combate à Lavagem de dinheiro e setores de inteligência, pesquisa e análise), com o intuito de explorar suas atuações e identificar possíveis soluções complementares, que contribuam com a agilidade de re- 
sultados, redução de tempo e custos para as instituições públicas no atendimento de demandas relacionadas a pesquisas, análises e investigações sobre o referido tema. 2) Do resultado do trabalho realizado no item 1 , foi criada uma proposta de método complementar de atendimento de demandas destinadas a esses laboratórios e setores especializados, resultante da identificação de dificuldades e necessidades dos mesmos.

3) Por fim, foi realizada uma experiência de uso em um Ministério Público Estadual com o intuito de comprovar a eficiência da proposta no atendimento das contribuições conforme item 1. A experiência foi realizada por intermédio da elaboração e implementação de um sistema de informação automatizado composto por diversas funcionalidades implementadas especificamente para o atendimento de algumas das diversas atividades demandadas encaminhadas aos setores e laboratórios especializados.

\section{AÇÕES E ESTRATÉGIAS NO COMBATE À CORRUPÇÃO E LAVAGEM DE DINHEIRO}

Com o passar do tempo, diversas instituições públicas brasileiras vêm adotando ações e medidas para obter maiores resultados, visando ao combate à corrupção, lavagem de dinheiro e criminalidade, de forma a reduzir os danos causados à sociedade e ao país.

Dentre elas, podemos citar a Estratégia Nacional de Combate à Corrupção e à Lavagem de Dinheiro (ENCCLA) que, contribuindo para a sistematização das várias iniciativas em torno dos temas da corrupção, e lavagem de dinheiro e para a articulação de diversos órgãos dos três poderes da República Brasileira, Ministérios Públicos e sociedade civil, foi criada em 2003 pelo Ministério da Justiça (ENCCLA, 2003).

Em suas ações, segundo Enccla (2003), a estratégia contribuiu com a geração de inúmeros resultados como, por exemplo, a criação do Programa Nacional de Capacitação e Treinamento para o Combate à Corrupção e à Lavagem de Dinheiro (PNLD), a estruturação do Grupo Nacional de Combate às Organizações Criminosas, no âmbito dos Ministérios Públicos Estaduais, a padronização da forma 
de solicitação/resposta de quebras de sigilo bancário, e respectivos rastreamentos e desenvolvimento do Sistema de Investigação de Movimentações Bancárias (SIMBA), e a criação do Laboratório de Tecnologia contra a Lavagem de Dinheiro, e replicação do modelo nas unidades da federação.

Outro exemplo de ações que podemos citar são as criações, e implantações de setores especializados de inteligência, pesquisa e análise, ações essas que costumam ser adotadas por órgãos de fiscalização, controle ou investigação como, por exemplo, os Tribunais de Contas, Ministérios Públicos e Polícias, em virtude de necessidade de setores especiais que visam à produção de conhecimento por via da utilização, conforme Almeida Neto (2009), de determinado procedimento metodológico e de técnicas específicas.

A nomenclatura dada a esses setores especializados costuma diferir de órgão para órgão, mas suas atividades costumeiramente demandadas, conforme cita França Junior (2001), são de coletas e cruzamento de dados de diversas fontes, análise de dados gerando conhecimento, e reportando-o por meio de relatórios diversos, normalmente nomeados como, por exemplo, de relatórios de inteligência, relatórios de análise ou relatórios técnicos.

Essas coletas, análises e geração de conhecimento obviamente terá sua complexidade e disposição de tempo e recursos, dependendo de cada demanda que pode demorar de dias a meses.

\subsection{Laboratório de Combate à Lavagem de Dinheiro (LAB-LD)}

Sendo instalado em 2007, segundo o Ministério da Justiça (2018), por intermédio de convênio entre o Ministério da Justiça e o Banco do Brasil, o Laboratório de Tecnologia contra a Lavagem de Dinheiro é resultado da meta 16 da Estratégia Nacional de Combate à Corrupção e à Lavagem de Dinheiro (ENCCLA), que previa a necessidade 
de implantar um laboratório modelo para a aplicação de soluções tecnológicas de analises de grandes volumes de informações, e para difusão de estudos sobre melhores práticas de uso de tecnologias e adequação de perfis profissionais.

A motivação para sua criação surgiu da observação de que investigações de casos de corrupção, ou lavagem de dinheiro, envolviam quebras de sigilo de um grande volume de contas bancárias, informações telefônicas e fiscais de longos períodos, o que gerava um grande volume de informações para ser analisada, onde ainda ocorria de muitas investigações e análises serem conduzidas por pessoas que não tinham a especialização técnica devida (MINISTÉRIO DA JUSTIÇA, 2018).

Segundo Pina (2014), o LAB-LD é uma unidade de análise de dados, que visa a identificação de atividades ilícitas mediante a aplicação de soluções tecnológicas, metodologias diversas, possuindo perfis profissionais próprios.

O trabalho em procedimentos persecutórios sobre a corrupção e lavagem de dinheiro desenvolvido nesses laboratórios especializados se desenvolve, basicamente, a partir de quatro tipos de análises, sendo elas: cadastral, fiscal, bancária e telefônica, onde a primeira costuma ser realizada em fontes, que o próprio órgão que implantou o LAB tem acesso, ou requisita a demais órgãos com os quais possui algum convênio, por exemplo, e as três últimas decorrem de quebras de sigilos autorizadas por ordem judicial (PINA, 2014).

Para a realização desses procedimentos, conforme cita Pina (2014), são utilizadas diversas tecnologias como, por exemplo, softwares especializados como Ferramentas I2 da IBM (International Business Machines), normalmente utilizado para cruzamentos de bases de dados, e geração de gráficos de vínculos, softwares de estatísticas, Microsoft Excel ou software de ETL (ferramentas para extração de dados de diversos sistemas, transformando esses dados conforme regras de negócio e, por fim, disponibilizando-os aos usuários). 


\section{PROPOSTA DO MODELO DE ATENDIMENTO ÀS DEMANDAS}

A proposta resultante desse estudo visa a disponibilizar uma solução complementar as duas principais abordagens selecionadas (LAB-LD e setores de inteligência, pesquisa e análise), e atualmente adotadas pelas instituições públicas no combate à corrupção, lavagem de dinheiro e outros crimes.

Na Figura 1, consta um exemplo ilustrativo de modelo do fluxo de demandas atualmente utilizado pelas instituições públicas, que possuem LAB-LD, ou setores de inteligência, pesquisa e análise.

\section{Figura 1 - Exemplo de fluxo geral}

\section{de demandas atualmente utilizado}

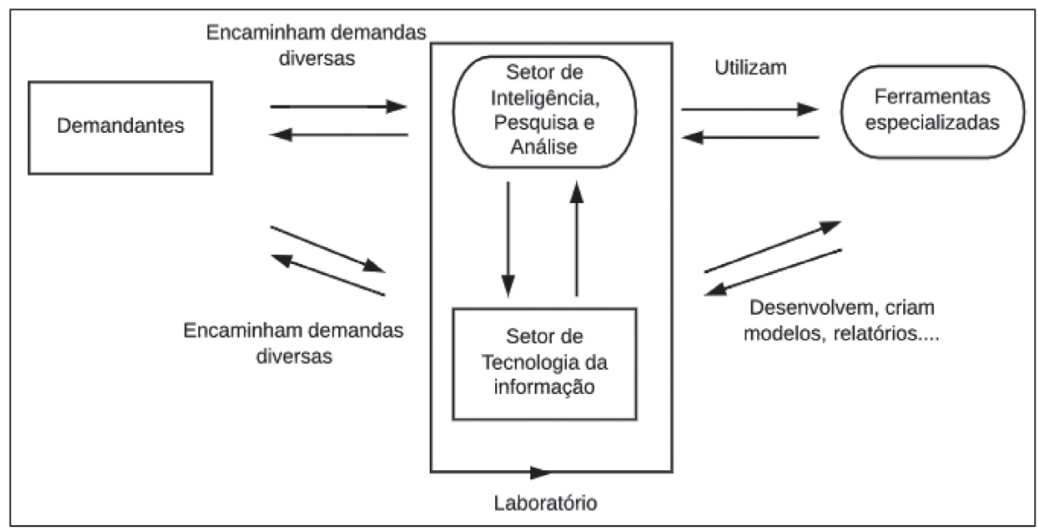

Fonte: Elaborado pelo próprio autor

No modelo geral acima apresentado, demandantes encaminham solicitações aos setores especializados, que utilizam ferramentas, ou softwares diversos, para o atendimento de demandas, nos quais retornam os resultados aos demandantes por meio de relatórios específicos dependendo de cada solicitação. 
Verifica-se também solicitações dos demandantes aos subsetores de tecnologia desses laboratórios especializados, ou solicitações dos próprios setores de inteligência aos subsetores de tecnologia que costumam dar suportes diversos para o atendimento das demandas.

Como já mencionado anteriormente, apesar dos excelentes resultados obtidos com o auxílio desses laboratórios e setores especializados, devido à alta demanda, falta de especialização ou conhecimento técnico, e limitações de recursos humanos existentes nos demandantes, ocorre um alto volume de demandas encaminhadas a esses laboratórios, acarretando em limitação quanto a seu atendimento.

Consequentemente, muitos setores de inteligência, pesquisa e análise ou LAB-LD, acabam definindo critérios para aceitação das demandas, ou seja, por não conseguirem atender o alto volume de solicitações, estabelecem requisitos mínimos para que seja aceito o recebimento de uma demanda pelo setor.

Como por exemplo, podemos citar um laboratório de tecnologia contra a lavagem de dinheiro, que realiza analises bancárias, fiscais e telefônicas ou consultas, e cruzamento de informações cadastrais de pessoas físicas e jurídicas, onde devido à limitação quantitativa de demandas que pode receber dentro de um determinado período, acaba tendo que definir requisitos mínimos para uma demanda, como por exemplo, para uma demanda de análise bancária, o laboratório pode definir que só aceita demandas desse assunto com um número mínimo de investigados, e demandas abaixo desse número teriam seu atendimento recusado.

Porém, como consequência, verifica-se que demandas que não atendem a esses requisitos mínimos, mas que ainda dependem de expertise técnica para o atendimento ou auxílio tecnológico existente nesses setores acabam tendo que ser atendidas pelos próprios demandantes que, por suas limitações, podem acarretar em atendimento ineficiente da demanda, ou até com erros.

Na Figura 2, verifica-se um exemplo de modelo de fluxo de aten- 
dimento de demandas complementar ao modelo atualmente utilizado, no qual acrescenta-se uma camada intermediária composta de sistemas automatizados entre os demandantes e laboratórios, ou setores especializados, nos quais possibilitam o atendimento de demandas básicas encaminhadas a esses setores e possíveis de serem automatizadas.

\section{Figura 2 - Exemplo de fluxo geral de demandas (Proposta)}

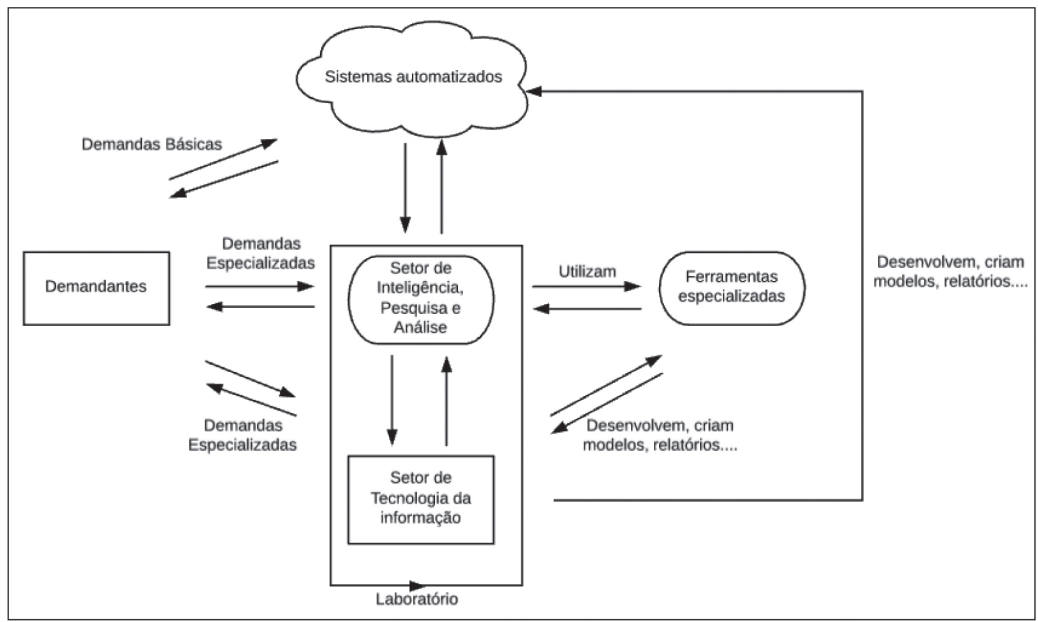

Fonte: Elaborado pelo próprio autor

A solução proposta possibilita não só a redução do número de demandas encaminhadas aos setores especializados, mas, também, maior agilidade de resultados e redução de custo e tempo para a realização de diversas atividades necessárias para o atendimento das solicitações.

Em um exemplo ilustrativo, e ainda dentro da demanda de uma análise bancária citada no exemplo anterior, um demandante, utilizando o sistema automatizado, iria simplesmente realizar uma solicitação ao sistema, e seria gerado um relatório automatizado com 
todas as informações dos investigados, com as transações bancárias realizadas entre eles, informações de depósitos não identificados e cheques, identificação de todas as pessoas físicas e jurídicas com as quais cada investigado transacionou, entre outros.

Assim, esses sistemas reduziriam, por exemplo, as solicitações de cruzamentos de transações bancárias que costumam demorar de dias a semanas para serem realizadas, focando apenas em solicitações mais especializadas para serem encaminhadas aos laboratórios.

Essa solução, dentro da realidade das instituições públicas brasileiras, já contribuiria, não só efetivamente para a redução de demandas e incremento de resultados mais ágeis, mas, também, possibilita disponibilizar aos demandantes que se encontram em situações com uma maior deficiência de recursos humanos, tecnológicos e de pessoas especializadas, oportunidades de atuação com aparato tecnológico de forma a, junto com a instituição como um todo, usufruir efetivamente das soluções tecnológicas que impactam no incremento de resultados.

Com isso, ocorre também a possibilidade de incremento de resultados com respectiva redução de tempo e custos, visto que, possibilitando um acesso direto aos demandantes a sistemas automatizados, que disponibilizam rapidamente os resultados de suas solicitações, consequentemente a sociedade será incrivelmente beneficiada com resultados mais rápidos e com redução de recursos públicos gastos para realizá-los.

\section{EXPERIÊNCIA DE USO E RESULTADOS}

Com o intuito de analisar a eficiência e viabilidade da proposta quanto à contribuição com a redução de demandas encaminhadas aos setores de inteligência, pesquisa e análise, assim como agilidade de resultados, entre outros benefícios, foi realizada uma experiência em um Ministério Público Estadual com base na proposta deste trabalho. 


\subsection{Configurações da experiência}

Para a realização da experiência, realizaram-se quatro ações específicas. Foram elas:

1) Identificação de dificuldades e necessidades dos órgãos do Ministério Público Estadual do Ceará, com foco inicial aos órgãos de investigação, visando à busca de soluções específicas para as demandas reais existentes, e que diminuam o tempo de trabalho para o atendimento das mesmas, automatizem diversas atividades, atuando na redução também de custos, e cujo acesso às soluções seja simples, com interface amigável e uso facilitado por todos os procuradores, promotores, servidores, entre outros atuantes na instituição.

2) Projeto e implementação de funcionalidades de acessos às bases de dados junto a diversos órgãos, que o Ministério Público Estadual selecionado tem convênio, ou acordo de cooperação técnica, para disponibilização das bases de dados de forma online e atualizada (preferivelmente), para que o sistema automatizado possa realizar a coleta, e cruzamento instantâneo de informações provenientes de diversas fontes, disponibilizando as mesmas em formulários, relatórios, gráficos diversos e gráficos de vínculos.

3) Elaboração e implementação de um sistema de informação, sendo o mesmo composto por diversos módulos de software (subsistemas), ou funcionalidades implementadas, especificamente, para diversas atividades de consulta e cruzamento de informações, possibilitando a geração de relatórios automatizados, entre outros.

4) Elaboração de manuais e tutoriais, divulgação e realização de treinamentos, para possibilitar uma utilização mais efetiva da solução implementada e por um número maior de usuários.

\subsection{Resultados e Discussões}

Enquanto muitas demandas estavam centralizadas nos setores de pesquisa, análise e inteligência (até mesmo demandas de consultas de informações sobre investigados) em virtude das limitações de tempo, recursos (humanos e tecnológicos) e alto número de demandas existentes nas diversas promotorias do Ministério selecionado, após 
a disponibilização do sistema automatizado, ocorreu, não só uma redução da quantidade de demandas encaminhadas a esses setores, como, também, contribuiu com a agilidade de resultados e serviços prestados por essas promotorias.

Os relatórios automatizados disponibilizados pelo sistema já passaram a ser parte integrante, e essencial de diversos processos dos órgãos do Ministério, principalmente os relatórios automatizados de coleta de informações de pessoas físicas e jurídicas, que são utilizados, não só pelos órgãos de investigação, mas, também, centros de apoio e demais áreas de atuação das promotorias da capital e interior.

Dentre as atividades realizadas pelos laboratórios e setores, que foram automatizadas com o intuito de possibilitar o acesso direto pelos demandantes, reduzindo o número de demandas a esses setores, podemos citar, por exemplo, atividades de consultas de informações sobre pessoas físicas e jurídicas investigadas, coleta e cruzamento de informações sobre licitações ocorridas nos municípios do estado, cruzamento de informações de transações bancárias entre investigados que tiveram a quebra de sigilo autorizada judicialmente, entre outras.

Com isso, verificou-se também uma maior agilidade, e simplificação na realização de diversas atividades de investigação, análise e coleta de informações, contribuindo para a melhoria da qualidade do trabalho, reduzindo custos e tempo para a instituição, e refletindo positivamente para a sociedade, onde, decorrido um período de um ano após a implantação inicial do sistema, ocorreu o reconhecimento de sua eficiência e importância pela instituição, ocorrendo o reconhecimento oficial pela alta gestão do Ministério Público do Estado do Ceará, e governador do respectivo estado (DOECE, 2017).

Em complemento às contribuições citadas, também podemos citar como pontos positivos: o fato de que as funcionalidades implementadas são criadas especificamente para o atendimento de demandas reais das instituições públicas que são implantadas; o fato de serem ferramentas gratuitas, não ocorrendo investimentos extras para a 
instituição que adotar a proposta; o modelo modular de sistema, onde possibilita a inclusão constante de novas funcionalidades no decorrer do surgimento de novas necessidades; e a mobilização de diversos demandantes contribuindo com novas ideias ou necessidade de inclusão de novas funcionalidades, ou bases de dados.

\section{CONSIDERAÇÕES FINAIS}

Este trabalho apresentou uma proposta para contribuir com estudos e ações voltadas à agilidade de resultados, e economia de recursos e tempo para a realização de atividades por instituições públicas brasileiras, referentes ao combate à corrupção, lavagem de dinheiro e demais crimes.

É importante ressaltar que a implementação da solução pode ser adaptada à realidade de cada órgão, assim como a relevância do assunto gera impactos, não só em instituições públicas brasileiras, mas, também, na sociedade como todo.

A proposta apresentada comprovou apresentar resultados promissores e reconhecidos pela instituição e estado onde foi implantada, onde para trabalhos futuros, sugere-se a realização de novas experiências de uso em demais estados, e órgãos que possuam laboratórios, ou setores especializados, assim como respectiva verificação e reconhecimento de resultados após sua implantação.

\section{A MODEL PROPOSAL FOR DEMANDING \\ INTELLIGENCE, RESEARCH AND ANALYSIS SECTOR OF BRAZILIAN PUBLIC INSTITUTIONS}

\section{ABSTRACT}

The evolution of information technology not only has an impact on the life of society, but also on the evolution and the way criminals 
have been acting. In this context, public institutions have been adopting measures to combat organized crime in Brazil, in which, among existing actions, we can mention the installation of Laboratories to Combat Money Laundering and creation of intelligence, research and analysis sectors. Despite the good results presented by these measures, unfortunately the demand for requests is high, which entails limitations in their treatment. This paper proposes to make available a complementary proposal of a model for meeting demands addressed to these sectors and laboratories, with the purpose of contributing to the agility in the attendance through the automation of operational procedures with reduction of costs for the institutions, and reduction of time for the various research and analysis activities.

Keywords: Corruption. Money laundry. Information systems.

\section{REFERÊNCIAS}

ALMEIDA NETO, Wilson Rocha de. Inteligência e contra-inteligência no Ministério Público. Belo Horizonte: Dictum, 2009. BRASIL. Constituição (1988). Constituição da República Federativa do Brasil. Brasília, DF: Senado Federal: Centro Gráfico, 1988.

DOECE. Ato de Homologação e Elogio da Medalha e Prêmio do Mérito Funcional. Diário Oficial [do Estado do Ceará], Fortaleza, CE. Série 3, Ano IX, n. 241. 27 Dez, 2017. p. 151-152.

ENCCLA. Estratégia Nacional de Combate à Corrupção e à Lavagem de Dinheiro, 2003. Disponível em: <http://enccla.camara.leg.br/>. Acesso em: $27 \mathrm{FeV}, 2018$.

FRANÇA JÚNIOR, F. F. ATIVIDADE DE INTELIGÊNCIA NO MINISTÉRIO PÚBLICO. Revista do MPRN , v. 1, p. 52, 2011.

MINISTÉRIO DA JUSTIÇA. Laboratório de Tecnologia Contra a Lavagem de Dinheiro, 2018. Disponível em: <http://www.justica.gov.br/sua-protecao/ lavagem-de-dinheiro/LAB-LD>. Acesso em: 20 Fev, 2018.

PAIVA, André Luiz de; GARCIA, André Spuri; ALCANTARA, Valderí de Castro. Disputas Discursivas sobre Corrupção no Brasil: Uma Análise Discursivo-Crítica no Twitter. Rev. adm. contemp.,Curitiba, v. 21,n. 5,p. 627-647, Out. 2017 . Disponível em: <http://www.scielo.br/scielo.php?script=sci_ 
arttext\&pid=S1415-65552017000500627\&lng=en\&nrm=iso $>$. Acesso em: 24 Fev, 2018. http://dx.doi.org/10.1590/1982-7849rac2017160163.

PINA, Christiana Bahia Andrade. Estratégia do Ministério da Justiça na Difusão das Técnicas de Combate à Lavagem de Dinheiro no Brasil. Conteúdo Juridico, Brasília-DF: 10 abr. 2014. Disponível em: <http://www. conteudojuridico.com.br/?artigos\&ver=2.47647>. Acesso em: 27 Fev. 2018. SAADI, Ricardo Andrade; MACHADO, Diogo de Oliveira. Os valores da corrupção: administração de bens apreendidos e confiscados. Rev. direito GV, São Paulo , v. 13,n. 2,p. 484-519 ago. 2017 . Disponível em <http://www. scielo.br/scielo.php?script=sci_arttext\&pid=S1808-24322017000200484\&ln g=pt\&nrm=iso > . acessos em 24 Fev. 2018. http://dx.doi.org/10.1590/23176172201719.

SECRETARIA DE SEGURANÇA PÚBLICA. Laboratórios se unem e identificam R\$ 44 Bi com indícios de lavagem de dinheiro no Brasil em uma década. 15 Jan, 2018. Disponível em: < https://www.policiacivil.ma.gov.br/ labld/?p=803>. Acesso em: 05 Mar. 2018.

TRANSPARENAIA INTERNACIONAL. Global Corruption Barometer: Brazil. 2013. Disponível em: <https://www.transparency. org/gcb2013/ country/?country=brazil>. Acesso em: 05 Mar. 2018.

Corruption Perceptions Index 2016. 2017. Disponível em: <https:// www.transparency. org/news/feature/corruption_perceptions_index_2016>. Acesso em: 05 Mar. 2018. 